New therapies

\title{
Maintenance pharmacological immunosuppressive strategies in renal transplantation
}

\author{
John P Vella, Mohamed H Sayegh
}

\begin{abstract}
Summary
Current maintenance immunosuppressive regimens for transplantation are based on three classes of drugs: corticosteroids, immunophilin-binding agents (eg, cyclosporin and tacrolimus), and antimetabolites (eg, azathioprine and mycophenolate). Drugs from the various classes inhibit the immune system at different points and are thus synergistic when used in combination.
\end{abstract}

Keywords: renal transplantation, immunosuppressants

Laboratory of Immunogenetics and Transplantation, Renal Division, Department of Medicine, Brigham \& Women's Hospital and Harvard Medical School, Boston, MA 02115, USA

JP Vella

MH Sayegh

Accepted 27 June 1996
The introduction of cyclosporin ${ }^{1}$ in the early 1980 s for clinical use in renal transplantation resulted in improved short-term graft survival by approximately $15 \%$ at one year. ${ }^{2}$ However, despite an $85-90 \%$ one year graft survival, ${ }^{3}$ longterm survival as measured by the half-life is unchanged at seven to eight years for randomly matched cadaver kidneys. This is the result of chronic, progressive loss of functioning renal tissue due mostly to antigen-dependent immunological factors. ${ }^{4}$ Currently used maintenance immunosuppressive agents may exacerbate this process due to their nephrotoxic potential. Furthermore, renal transplant recipients are at risk of significant side-effects of immunosuppression including infection, ${ }^{5}$ cardiovascular disease and hypertension ${ }^{6}$ and malignancy. ${ }^{7}$ These limitations constitute part of the rationale for the continued development of new immunosuppressive agents with greater specificity and reduced toxicity. Several new drugs are emerging from the laboratory into clinical trials. ${ }^{8}$ In this paper we will discuss drugs presently in use in clinical practice and the status of the new drugs that are undergoing testing currently.

\section{Current immunosuppressive strategies}

\section{STEROIDS}

Corticosteroids remain one of the cornerstones of most induction, maintenance and rescue immunosuppressive regimens. Steroids are lympholytic when given in high dosage (eg, methylprednisolone, $1 \mathrm{~g} /$ day) and can reverse acute rejection in up to $70 \%$ of cases. ${ }^{9}$ Other effects include inhibition of complement activation and suppression of macrophage secretion of IL-1, which in turn suppresses secretion of IL-2 by activated T cells. There is no consensus on dosing protocols, however, most programmes institute high-dose induction therapy, followed by a gradual taper to achieve a maintenance dose of $5-15 \mathrm{mg}$ of prednisolone at $6-12$ months. The lack of specificity of the effects of steroids on the immune system, coupled with the vast array of serious side-effects makes steroid withdrawal an attractive objective, which is achievable in up to $80 \%$ of selected patients. ${ }^{10}$ Steroids should not be withdrawn until at least six months have elapsed since transplantation in the absence of rejection, or six months after the most recent rejection episode. Further, renal function should be stable as evidenced by a serum creatinine of less than $222 \mu \mathrm{mol} / 1(2.5 \mathrm{mg} / \mathrm{dl})$. Factors which predict failure of withdrawal include: withdrawal before six months, black race, donor/recipient racial mismatch and poor renal function. Steroid withdrawal results in favourable blood pressure responses, improvements in glycaemic control in post-transplantation diabetes mellitus, improvement in hyperlipidaemia, and perhaps reduces the risk of infection. ${ }^{10}$ Withdrawal should only be attempted in patients experiencing serious side-effects who satisfy the selection criteria listed above. When indicated, steroids should always be tapered very slowly, both to prevent acute rejection and preclude hypoadrenalism.

\section{AZATHIOPRINE (IMURAN)}

Azathioprine is an imidazolyl derivative of mercaptopurine and functions as an antimetabolite. It is well absorbed from the gastrointestinal tract and is metabolised in the liver to mercaptopurine. The active substance is further metabolised by xanthine oxidase, thus concordant treatment with allopurinol, an inhibitor of xanthine oxidase, can rapidly lead to toxic levels. Consequently, transplant recipients suffering from hyperuricaemia and gout, a not infrequent complication of cyclosporin, should have at least a $50 \%$ dose reduction in azathioprine if allopurinol is prescribed. After antigenic stimulation, nucleic acid synthesis in activated $T$ and $B$ cells increases dramatically, however, the purine analogues resulting from azathioprine 


\begin{tabular}{|l|}
\hline Cyclosporin drug \\
interactions: drugs that \\
decrease cyclosporin levels \\
\hline Well substantiated \\
- anticonvulsants: carbamazepine, \\
phenobarbitone, phenytoin \\
- antituberculous agents: rifampicin, \\
isoniazid \\
Isolated reports \\
- heparin \\
- metoprolol \\
- nafcillin \\
- pctreotide \\
- sodium volon \\
- sulpha-containing compounds \\
- sulfinpyrazone \\
- warfarin \\
\hline
\end{tabular}

Box 1

\begin{tabular}{|l|}
\hline Cyclosporin drug \\
interactions: drugs that \\
increase cyclosporin levels \\
\hline Well substantiated \\
- bromocriptine \\
- antifungals, including fluconazole, \\
itraconazole, ketoconazole \\
- danazol \\
- metoclopramide \\
- erythromycin \& clarithromycin \\
- verapamil, diltiazem \\
- methylprednisolone \\
Isolated reports \\
- acetazolamide \\
- antibiotics: amikacin, tobramycin, \\
framycetin, imipenem, \\
metronidazole, co-trimoxazole, \\
vancomycin \\
- cimetidine \\
oral contraceptives: oestradiol, \\
levonorgestrel/oestradiol, norethin- \\
drone/norethisterone \\
- warfarin \\
- nifedipine \\
- sulindac \\
\hline
\end{tabular}

Box 2

\begin{tabular}{|l|}
\hline Side-effects of cyclosporin \\
\hline - renal dysfunction (acute and \\
chronic) \\
- hyperkalemia and acidosis (normal \\
anion gap) \\
- hyperuricaemia and gout \\
- hypophosphataemia \\
- hyperlipidaemia \\
- infection \\
- tremor \\
- gingival hyperplasia \\
- hirsutism \\
- habetes mellitus \\
thrombolytic uraemic syndrome/ \\
purpura \\
\hline
\end{tabular}

Box 3 administration are cytotoxic and prevent $T$ and $B$ cell activation. The effect is non-specific and both cellular and humoral immunity pathways are affected. Leukopenia is the commonest side-effect of azathioprine therapy and is dose related. The immunosuppressive effects, however, are not dependent on total depletion of white cells. Azathioprine therapy can also be complicated by anaemia and thrombocytopenia and the effect is potentiated by antilymphocyte antibody therapy. ${ }^{11}$ Another rare, though potentially serious, side-effect is hepatotoxicity which manifests as abnormal liver function tests with cholestasis. Azathioprine has been linked with malignancy, especially cutaneous squamous and basal cell carcinomas. ${ }^{12}$ Unfortunately, this effect is not restricted to azathioprine as there is evidence to show that cyclosporin can also induce similar cutaneous changes. ${ }^{12}$ Consequently, renal transplant recipients should be advised to minimise exposure to the sun and/or use heavy sun blocks when they do.

\section{CYCLOSPORIN (SANDIMMUNE)}

Cyclosporin is a fat-soluble cyclic peptide antibiotic derived from the fungus Tolypocladium inflatum. ${ }^{1}$ Initially discovered in 1970, its immunosuppressive properties were discovered two years later and it became widely used in transplantation in the early 1980s. Cyclosporin blocks the calcium-dependent T-cell receptor signal transduction pathway by binding to cyclophilin, a cytoplasmic protein member of the immunophilin family. Although this binding inhibits the peptidylprolyl isomerase activity of cyclophilin, this is not the mechanism of inducing immunosuppression. Rather, the complex inhibits calcineurin, which is necessary for the dephosphorylation of cytosolic NF-AT (nuclear factor of activated $\mathrm{T}$ cells) prior to its translocation to the nucleus, where it binds to the IL-2 promoter sequence. The overall effect is to prevent transcription of IL-2 RNA and thus the inhibition of IL-2 secretion which is necessary for further $T$ cell activation and proliferation. As such, it has greater specificity for cell-mediated immune mechanisms than either steroid or azathioprine.

Cyclosporin is absorbed from the upper small intestine in a variable manner, resulting in an initial bioavailability of between 4 and $26 \% .{ }^{13}$ This can increase up to $57 \%$ after a period of weeks. ${ }^{14}$ Being lipid soluble, the volume of distribution is large and depends to some extent on the percentage body fat of the patient. Metabolism occurs via the hepatic cytochrome P450 system and results in approximately 15 metabolites in humans, many of which are active. Excretion is via the biliary tract. The half-life ranges from 14 to 27 hours. All of these factors, plus the various drug interactions that can occur (boxes 1 and 2), can lead to difficulties with maintaining adequate, non-toxic levels. The penalty for failure is rejection or cyclosporin-induced nephrotoxicity.

Cyclosporin nephrotoxicity can manifest both as functional and structural changes. The potent vasoconstriction seen after the administration of cyclosporin is not a direct effect, but is mediated both by stimulation of endothelin secretion, a highly vasoactive and mitogenic peptide, ${ }^{15}$ and also reduction in vasodilatory prostaglandins and nitric oxide. This can further lead to systemic hypertension as well as acutely decreasing the glomerular filtration rate by reducing renal plasma flow. Further metabolic affects can be caused by disturbances in tubular function leading to acidosis, hyperkalaemia, hypophosphataemia and hyperuricaemia. The factors responsible for chronic nephrotoxicity are not well understood. The primary lesion would appear to be vascular in nature with the development of endothelial damage and obliterative vasculopathy. This results in secondary ischaemia and ultimately, the development of interstitial fibrosis. This side-effect is well documented in recipients of heart and liver transplants as well as patients with auto-immune disease who are treated with cyclosporin. Whether there is a 'safe' dose of cyclosporin that is effective immunologically but does not cause progressive renal dysfunction is unknown. Uncontrolled studies indicate that using daily doses in the region of $3-5 \mathrm{mg} / \mathrm{kg}$ is associated with stable renal function. Potential side-effects of cyclosporin are summarised in box 3.

Recently, the manufacturers (Sandoz) have formulated a microemulsion preparation, Neoral, which is undergoing testing at present. This promises to deliver the drug in a more reliable fashion, giving rise to improvements in steady-state peak concentrations by up to $60 \% .{ }^{16}$ In turn, dosage reduction will perhaps be possible, though cynics maintain that costs will not be reduced as generic formulations of cyclosporin, if introduced when current patents expire, are less likely to be used by clinicians in the presence of a formulation with an improved pharmacokinetic profile. Currently, most patients are being maintained on triple therapy immunosuppression protocols with cyclosporin, steroids and azathioprine, although some centres use double therapy with 
cyclosporin and steroids. Monotherapy with cyclosporin is popular in some European centres. Concerns about the long-term nephrotoxic potential of cyclosporin need to be balanced against the risk of rejection. Withdrawal of cyclosporin is associated with an increased incidence of late rejection and a possible decrease in long-term graft survival, ${ }^{17,18}$ especially in black patients. ${ }^{19}$

\section{Emerging immunosuppressive strategies}

TACROLIMUS (FK506, PROGRAF)

Tacrolimus is a macrolide antibiotic which possesses similar, though more potent, immunosuppressant properties than cyclosporin on a milligram to milligram basis. The mechanism of action is similar to that of cyclosporin. Tacrolimus binds to an immunophillin, FKBP (FK506-binding proteins), which also have peptidylprolyl isomerase activity. Similar to cyclosporin, the immunosuppressive action is not mediated through loss of enzyme function of the complex, rather, blockade of calcineurin-mediated T-cell receptor signal transduction and inhibition of IL-2. It is approved by the US Food \& Drug Administration (FDA) for use in liver transplantation and is being widely used in this setting. It has the advantage of being more water soluble than cyclosporin and thus is less dependent on bile salts for absorption, and maintenance of therapeutic levels is less problematic. Its possible superiority over cyclosporin in renal transplantation is currently being evaluated.

Tacrolimus has a similar toxicity profile to cyclosporin. Both short- and long-term studies in liver transplant recipients suggest that it is at least as nephrotoxic as cyclosporin. ${ }^{20}$ In addition, long-term trials have found a similar incidence of acute renal failure, late hypertension and late renal insufficiency, although the latter problem may be slightly more prevalent with tacrolimus than cyclosporin. Tacrolimus can also cause hyperkalaemia, hyperuricae$\mathrm{mia}^{20,21}$ and, rarely, the haemolytic uraemic syndrome (compare with sideeffects of cyclosporin summarised in box 3). One potential benefit of tacrolimus in renal transplantation is as rescue therapy in patients with recurrent or resistant rejection on cyclosporin. ${ }^{22}$ One report, for example, switched 77 patients with biopsy-proven ongoing acute renal allograft rejection from cyclosporin to FK506. ${ }^{22}$ The overall response rate was $74 \%$, with responders having a mean plasma creatinine concentration of $207 \mu \mathrm{mol} / 1$ at 14 months. Even dialysis-dependent patients had a $50 \%$ response rate. Controlled trials and more prolonged follow-up are required before switching to tacrolimus can be recommended for these patients.

\section{MYCOPHENOLATE MOFETIL [RS-61443, CELLCEPT]}

Mycophenolate is a morpholinoethyl ester of mycophenolic acid. It inhibits the synthesis of guanosine monophosphate via blockade of the enzyme inosine monophosphate dehydrogenase. The drug blocks proliferative responses of $\mathrm{T}$ and $\mathrm{B}$ lymphocytes, and inhibits antibody formation and the generation of cytotoxic $T$ cells. It is not nephro- or hepato-toxic and may have less bone marrow toxicity than azathioprine. Gastrointestinal side-effects are commonplace, however, are dose related, and usually manifest as gastritis and diarrhoea. The role of mycophenolate as adjunctive therapy to current cyclosporin-based regimens in place of azathioprine is being evaluated. A recent randomised, double-blind, multicentre study performed in the US comparing azathioprine with mycophenolate revealed a significantly reduced risk of biopsy-proven acute rejection from $47.6 \%$ (azathioprine group) to $31 \%$ (mycophenolate group). ${ }^{23}$ Mycophenolate was also shown to significantly prolong the time to first biopsyproven rejection and reduced the necessity for anti-rejection therapy from $44.5 \%$ to $24.8 \%$. A European trial comparing cyclosporin and steroid therapy with that of mycophenolate or placebo demonstrated a reduction in the incidence of acute rejection by up to $26 \% .{ }^{24}$ Patients receiving the higher dose (ie, $3 \mathrm{~g}$ vs $2 \mathrm{~g}$ daily) benefited most with regard to prevention of rejection, although they tended to have a higher incidence of gastrointestinal, haematological and infectious complications.

Mycophenolate has recently been approved by the FDA solely for the prophylaxis of renal transplant rejection. Preliminary data on the role of mycophenolate as rescue therapy for biopsy-proven acute rejection revealed a response rate of $69 \%$ in patients who failed standard pulse steroid or OKT3 ${ }^{25}$ In addition, there is experimental evidence pointing to the role of mycophenolate in the management of chronic renal allograft rejection in rats. ${ }^{26}$ These preliminary data clearly require substantiation. If substantiated, however, they offer a glimmer of hope for what is the major problem in clinical transplantation today. 
- new formulations of cyclosporin

- mycophenolate [RS 61443]

- rapamycin

\section{Emerging immunosuppres- sant agents in renal transplantation}

- tacrolimus [FK 506]

RAPAMYCIN (SIROLIMUS)

Rapamycin is a macrolide antibiotic produced by a fungus Streptomyces hygroscopicus found in Easter Island. Being structurally similar to tacrolimus, it is not surprising that rapamycin binds to the same class of cytoplasmic proteins, the immunophilins. While cyclosporin and tacrolimus inhibit calcineurin-dependent $\mathrm{T}$-cell receptor signal transduction (commonly known as signal 1), rapamycin blocks transduction of the co-stimulatory CD28 signal (signal 2) by inhibiting the phosphorylation of $\mathrm{p} 70^{\text {s6 }}$ kinase which is necessary for full T-cell activation. ${ }^{27}$ Although rapamycin binds to the same FKBP that tacrolimus binds to, it results in inhibition of response to cytokines rather than inhibition of secretion of cytokines. There is laboratory evidence to suggest that, due to competitive binding, rapamycin may work antagonistically with tacrolimus. Despite relatively poor absorption, adequate immunosuppression is achieved with levels in the picomolar range. Rapamycin is 10 times more potent than cyclosporin and four times more potent than tacrolimus. Sideeffects include gastrointestinal toxicity with musosal necrosis and submucosal vasculitis reported in animal models (usually dogs). Thrombocytopenia and dyslipidaemia have been seen in phase I studies. In humans, rapamycin is being developed as a maintenance immunosuppressive drug to be used in combination with cyclosporin. Some preliminary phase II studies suggest a possible reduction in the dose of cyclosporin or steroid tapering when rapamycin is used concomitantly. There is also evidence suggesting a role for rapamycin in the long-term prevention of chronic rejection in animal models. ${ }^{28}$ Whether this important observation is confirmed in humans by long-term studies remains to be determined. Phase II-III clinical trials are currently underway.

\section{Trends for the future}

Given the current lack of consensus on the optimum regimen for immunosuppression of renal transplant recipients with our existing array, it is likely that new drugs, as they are approved, will cloud the issues further initially. The important properties to be sought for in any new treatment are: greater specificity, decreased side-effects, and greater efficacy in the management of chronic rejection. The combination of effective antiproliferative activity as exhibited by these newer agents and the well-established effect of cyclosporin on $T$-cell activation offers the potential for the development of new regimens which fulfill at least some of these criteria.

The holy grail of transplantation is the induction of tolerance, by which we mean specific acceptance of allograft tissue without immunosuppression in the presence of an intact humoral and cell-mediated immune system. ${ }^{29}$ Clinical strategies of tolerance have been investigated with the use of blood transfusion or donor bone marrow infusion. ${ }^{30}$ Currently, we and others are actively researching the implications of $\mathrm{T}$-cell activation blockade by a variety of different mechanisms. One strategy is to interfere with T-cell recognition of alloantigen by blocking the peptide/T-cell receptor signal and thus abrogate the antigen-specific component of the immune response. ${ }^{30,31}$ Another strategy is to interfere with various co-stimulatory pathways that are also necessary for full $\mathrm{T}$ cell activation and thus induce antigen-specific unresponsiveness. ${ }^{30,32}$ Some of these strategies may undergo clinical evaluation in the near future. Finally, the effect of the interactions between pharmacological agents currently in use and the novel tolerising agents under development remains to be established. Animal studies suggest that, although cyclosporin may inhibit tolerance induction, rapamycin may work synergistically. Further work remains to be done before a definitive statement can be made on this topic.

8 Halloran PF. New trends in immunosuppression for renal transplantation. Curr Opin Nephrol Hypertens 1994; 6: 575-7

1 Kahan BD. Drug therapy: cyclosporine. $N$ Engl $f$ Med 1989; 321: 1725-38

2 Cecka JM, Terasaki PI. The UNOS scientific data registry. In: Terasaki PI, ed. Clinical transplants. Los Angeles: UCLA Tissue Typing Laboratory, 1993; p 1.

3 Terasaki PI, Cecka JM, Cho Y. Overview. In: Terasaki PI ed. Clinical transplants. Los Angeles: UCLA Tissue Typing Laboratory, 1989; p 585.

4 Carpenter CB. Long term failure of renal transplants: adding insult to injury. Kidney Int transplants: addin:

5 Rubin RH. Infectious complications of renal transplantation. Kidney Int 1993; 44: 221-36. 6 Braun WE. Long-term complications of renal transplantation [clinical conference]. Kidney In 1990; 37: $1363-78$

7 Penn I. Cancers complicating organ transplantation. N Engl $f$ Med 1990; 323: 1767-9.
9 Gray D, Shepherd H, Daar A, et al. Oral versus intravenous high dose steroid treatment of renal transplant rejection. Lancet 1978; 1: 117-8

10 Hricik DE, Almawi WY, Strom TB. Trends in the use of glucocorticoids in renal transplantathe use of glucocorticoids in renal tran
tion. Transplantation 1994; 57: 979-89.

11 Heaf J. Bone marrow suppression during quadruple immunosuppressive therapy after renal ruple immunosuppressive therapy after renal

12 Gupta AK, Cardella CJ, Haberman HF. Cutaneous malignant neoplasms in patients with neous malignant neoplasms in patients with
renal transplants. Arch Dermatol 1986; 122: renal trans $1288-93$.

13 Kahan BD, Ried M, Newburger J. Pharmacokinetics of cyclosporin in human renal transplantation. Transplant Proc 1983; 15: 466-53.
14 Kahan BD. Cyclosporine: a powerful addition to the immunosuppressive armamentarium. $A m \mathcal{J}$ Kidney Dis 1984; 3: 444-55.

15 Watschinger B, Sayegh MH. Endothelin in organ transplantation. Am $\mathcal{F}$ Kidney Dis 1996; 27: $151-61$.

16 Kovarik JM, Mueller EA, van Bree JB, et al. Cyclosporine pharmacokinetics and variability Cyclosporine pharmacokinetics and variability from a micromulsion formulation - a multicenter investigation in kidney transplant pa-
tients. Transplantation 1994; 58: 658-63.

17 Dunn J, Golden D, Van Buren CT, et al. Causes of graft loss beyond 2 years in the cyclosporine

18 Heim-Duthoy KL, Chitwood KK, Tortorice year after renal transplantation. Am $\mathcal{F}$ Kidney Dis 1994; 24: 846-53. of graft loss beyond 2 years in the cyclosp $\mathrm{KL}, e t$ al. Elective cyclosporine withdrawal 1 
19 Sanders CE, Curtis JJ, Julian BA, et al. Tapering or discontinuing cyclosporine for financial reasons - a single centre experience. Am $\mathcal{f}$ Kidney Dis 1993; 21: 9-15.

20 The US Multicenter FK506 Liver Study Group. A comparison of tacrolimus and cyclosporine for immunosuppression in liver transplantation. $N$ Engl f Med 1994; 331: 1110-5.

21 Porayko MK, Textor SC, Krom RA, et al. Nephrotoxic effects of primary immunosuppression with FK-506 and cyclosporine regimens after liver transplantation. Mayo Clin Proc 1994; 69: $105-11$.

22 Jordan ML, Shapiro R, Vivas SA, et al. FK506 'rescue' for resistant rejection of renal allografts under primary cyclosporine immunosuppression. Transplantation 1994; 57: 860-5.

23 US Renal Transplant Mycophenolate Mofetil Study Group. Mycophenolate mofetil for the prevention of acute rejection in primary cadaveric renal allograft recipients. Transplantation 1995; 60: 225-32.
24 European Mycophenolate Mofetil cooperative study group. Placebo-controlled study of mycophenolate mofetil combined with cyclosporin and corticosteroids for prevention of acute rejection. Lancet 1995; 345: 1321 - 5 .

25 Sollinger HW, Belzer FO, Deierhoi MH, et al. RS-61443 (mycophenolate mofetil). A multicenter rejection. Ann Surg 1992; 216: 513-8.

26 Azuma H, Binder J, Heemann U, et al. Effect of RS-61443 on chronic rejection of rat kidney allografts. Transplant Proc 1995; 27: 436-7.

27 Chung J, Kuo CJ, Crabtree GR, Blenis J. Rapamycin-FKBP specifically blocks growth dependent activation of and signalling by the 70kd S6 protein kinases. Cell 1992; 69: $1227-$ 36.
28 Morris RE. Rapamycins: antifungal, antiproliferative and immunosuppressive macrolides. Transplant Rev 1992; 6: 39.

29 Nickerson PW, Stenrer W, Steiger J, Strom TB. In pursuit of the 'Holy Grail'; allograft tolerance. Kidney Int 1994; 45:s40-49.

30 Turka LA, Sayegh MH. T Cell tolerance. In: Tilney NL, Strom TB, Paul LC, eds. Transplantation biology: cellular and molecular aspects. New tation biology: cellular and molecular aspects.

31 Sayegh MH, Krensky AM. Novel immunotherapeutic strategies using MHC derived peptides. apeutic strategies using MHC derived pept

32 Sayegh MH, Turka LA. T cell costimulatory pathways: promising novel targets for immunosuppression and tolerance induction. $\mathcal{f} \mathrm{Am}$ Soc Nephrol 1995; 6: 1143-50.

\section{Grants for travel and research 1997}

The Fellowship of Postgraduate Medicine is offering grants for travel, or towards the cost of equipment, for young graduates entering careers in clinical research. These are intended to 'top-up' grants for existing research, or for specific purposes of travel in connection with the research, and are of the order of $£ 1000$.

The application should give a concise summary of the research project, the sum requested, details of the equipment or of the travel project, and include a curriculum vitae of the applicant.

Successful applicants will be expected to submit reports of their research for publication in the Postgraduate Medical fournal.

Applications should be sent to:

Mrs E J Young

Executive Secretary

The Fellowship of Postgraduate Medicine

12 Chandos Street

London W1M 9DE, UK

CLOSING DATE FOR APPLICATIONS: 30 November 1997 